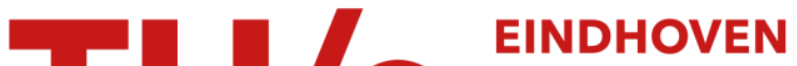 UNIVERSITY OF TECHNOLOGY
}

\section{Structural transitions and arrest of domain growth in sheared binary immiscible fluids and microemulsions}

\section{Citation for published version (APA):}

Harting, J. D. R., Giupponi, G., \& Coveney, P. V. (2007). Structural transitions and arrest of domain growth in sheared binary immiscible fluids and microemulsions. Physical Review E - Statistical, Nonlinear, and Soft Matter Physics, 75(4), 041504-1/12. [041504]. https://doi.org/10.1103/PhysRevE.75.041504

DOI:

10.1103/PhysRevE.75.041504

Document status and date:

Published: 15/01/2007

\section{Document Version:}

Publisher's PDF, also known as Version of Record (includes final page, issue and volume numbers)

\section{Please check the document version of this publication:}

- A submitted manuscript is the version of the article upon submission and before peer-review. There can be important differences between the submitted version and the official published version of record. People interested in the research are advised to contact the author for the final version of the publication, or visit the $\mathrm{DOI}$ to the publisher's website.

- The final author version and the galley proof are versions of the publication after peer review.

- The final published version features the final layout of the paper including the volume, issue and page numbers.

Link to publication

\section{General rights}

Copyright and moral rights for the publications made accessible in the public portal are retained by the authors and/or other copyright owners and it is a condition of accessing publications that users recognise and abide by the legal requirements associated with these rights.

- Users may download and print one copy of any publication from the public portal for the purpose of private study or research.

- You may not further distribute the material or use it for any profit-making activity or commercial gain

- You may freely distribute the URL identifying the publication in the public portal.

If the publication is distributed under the terms of Article 25fa of the Dutch Copyright Act, indicated by the "Taverne" license above, please follow below link for the End User Agreement:

www.tue.nl/taverne

Take down policy

If you believe that this document breaches copyright please contact us at:

openaccess@tue.nl

providing details and we will investigate your claim. 


\title{
Structural transitions and arrest of domain growth in sheared binary immiscible fluids and microemulsions
}

\author{
J. Harting, ${ }^{1}$ G. Giupponi, ${ }^{2}$ and P. V. Coveney ${ }^{2}$ \\ ${ }^{1}$ Institute for Computational Physics, University of Stuttgart, Pfaffenwaldring 27, 70569 Stuttgart, Germany \\ ${ }^{2}$ Centre for Computational Science, Department of Chemistry, University College London, \\ 20 Gordon Street, WC1H OAJ London, United Kingdom \\ (Received 15 January 2007; published 26 April 2007; publisher error corrected 27 April 2007)
}

\begin{abstract}
We investigate spinodal decomposition and structuring effects in binary immiscible and ternary amphiphilic fluid mixtures under shear by means of three-dimensional lattice Boltzmann simulations. We show that the growth of individual fluid domains can be arrested by adding surfactant to the system, thus forming a bicontinuous microemulsion. We demonstrate that the maximum domain size and the time of arrest depend linearly on the concentration of amphiphile molecules. In addition, we find that for a well-defined threshold value of amphiphile concentration, the maximum domain size and time of complete arrest do not change. For systems under constant and oscillatory shear we analyze domain growth rates in directions parallel and perpendicular to the applied shear. We find a structural transition from a sponge to a lamellar phase by applying a constant shear and the occurrence of tubular structures under oscillatory shear. The size of the resulting lamellae and tubes depends strongly on the amphiphile concentration, shear rate, and shear frequency.
\end{abstract}

DOI: 10.1103/PhysRevE.75.041504

PACS number(s): 64.75.+g, 47.11.-j, 82.70.Uv, 83.60.-a

\section{INTRODUCTION}

Complex fluid mixtures consisting of immiscible fluid species and surfactants are ubiquitous in many industrial applications. These fluid mixtures involve both hydrodynamic flow effects and complex interactions between fluid particles. Typical examples can be found in the food, cosmetic, and chemical industries where surfactants are utilized to stabilize otherwise immiscible fluids. A good example is a barbecue sauce containing large fractions of water and oil or fat. Without any additives the constituents would phase separate, entering a not very appealing demixed state in which the fat accumulates in a thick layer on top of the remainder. Adding an emulsifier or surfactant helps to stabilize the sauce. These molecules are often called amphiphiles and, in their simplest form, are comprised of a hydrophilic (water-loving) head group and a hydrophobic (oil-loving) tail. The surfactant molecules self-assemble on the surface of oil droplets and reduce the surface tension. Thus, the droplets stabilize and remain suspended within the bulk water. A typical emulsifier used by the food industry is egg yolk lecithin. Proteins and emulsifiers with low molecular weight are also common emulsifiers.

Amphiphilic fluids containing at least one species made of surfactant molecules are in general complex fluids that can self-assemble to form ordered structures such as lamellae, micelles, not ordered sponge phases and liquid crystalline (cubic) phases. In general, adding amphiphiles to a binary mixture of otherwise immiscible fluids (for example oil and water) that is undergoing phase separation can cause the demixing process to slow down. If the amphiphile concentration is sufficiently high, the demixing process might eventually arrest completely. It has been shown by Langevin, molecular dynamics, lattice gas, and lattice Boltzmann simulations that the temporal growth law for the size of oil and water domains in a system without amphiphiles follows a power law $t^{\alpha}[1,2]$ and crosses over to a logarithmic growth law $(\ln t)^{\theta}$, where $\alpha, \theta$ are fitting parameters and $t$ is the time [3-5]. A further increase of the surfactant concentration can lead to growth which is well described by a stretched exponential form $A-B \exp \left(-C t^{D}\right)$, where capital letters denote fitting parameters $[4,5]$. By adjusting temperature, fluid composition, or pressure, amphiphiles can self-assemble and force the fluid mixture into a number of equilibrium structures or mesophases. These include lamellae and hexagonally packed cylinders, micellar, primitive, diamond, or gyroid cubic mesophases as well as sponge phases. In this paper we focus on the sponge mesophase, which in the context of our simulations is called a bicontinuous microemulsion since it is formed by the amphiphilic stabilization of a phase-separating binary mixture, where the immiscible fluid constituents occur in equal proportions. Here, the oil and water phases interpenetrate and percolate and are separated by a monolayer of surfactant at the interface.

Such complex fluids are often subject to shear forces and show pronounced rheological properties [6,7]. Often, shearinduced transitions from isotropic to lamellar phases can be observed. These have been studied experimentally in binary [8] and ternary amphiphilic fluids $[9,10]$. If oscillatory shear is applied, a further transition to a tubular phase or a transition between differently oriented lamellar phases can occur [11-14].

Computationally, such complex fluids are too large and expensive to tackle with atomistic methods such as molecular dynamics, yet they require too much molecular detail for continuum Navier-Stokes approaches. Algorithms that work at an intermediate or "mesoscale" level of description have been developed during the last twenty years, including dissipative particle dynamics [15-17], lattice gas cellular automata [18], the stochastic rotation dynamics [19-21], and the lattice Boltzmann equation [22-24]. In particular, the lattice Boltzmann method has been found highly useful for simulating complex fluid flows in a wide variety of systems. This algorithm, described in more detail in the next section, 
is extremely well suited to implementation on parallel computers, which permits very large systems to be simulated, reaching hitherto inaccessible physical regimes.

In this paper we investigate spinodal decomposition and structuring effects in binary immiscible and ternary amphiphilic fluid mixtures under shear by means of large scale three dimensional lattice Boltzmann (LB) simulations. The purely kinetic LB method we use is able to model complex flows whose rheological properties are emergent from the mesoscopic kinetic processes without any imposed macroscopic constraints [25].

Varieties of the lattice Boltzmann method have been used successfully to study the behavior of multiphase flows in the past. A number of authors have investigated spinodal decomposition [2,26-36] and the same phenomenon has also attracted some interest in the presence of shear, where structural transitions from isotropic to lamellar or tubular phases may occur $[14,37-40]$.

There have been only limited investigations of the influence of amphiphiles on the domain growth within the lattice Boltzmann method, despite the fact that ternary amphiphilic fluids have been studied by a number of authors [24,41-43]. For example, it has been shown that the lattice Boltzmann method can be used to describe the self-assembly and the rheological properties of mesophases including the primitive $P$ phase [44] and the gyroid phase [45]. The gyroid mesophase in particular has been of major interest during the last few years, where the phase formation and structural properties [5,46], the influence of defects [47-50], as well as its properties under shear $[45,51]$ have been investigated.

In this paper, our aim is to focus on the effect of surfactant concentration on the length and time scales of arrested growth and on the changes in structural properties induced by steady or oscillatory shear.

The remainder of the paper is organized as follows. After an introduction to the simulation method and the fluid parameters used, we present results from the simulation of ternary amphiphilic systems with varying surfactant density. Here, we draw on the results of González-Segredo et al. [5] for the simulation parameters used within the present work. Thereafter, we extend our study to systems under constant and oscillatory shear, where we report on the influence of the domain growth rates and structural transitions induced by the shear. Finally, a summary and conclusions are given.

\section{SIMULATION METHOD}

A set of equations can be used to represent a standard LB system involving multiple species [52]

$$
n_{i}^{\alpha}\left(\mathbf{x}+\mathbf{c}_{i}, t+1\right)-n_{i}^{\alpha}(\mathbf{x}, t)=\Omega_{i}^{\alpha}
$$

with $i=0,1, \ldots, b$. The single-particle distribution function $n_{i}^{\alpha}(\mathbf{x}, t)$ indicates the density of species $\alpha$, having velocity $\mathbf{c}_{i}$, at site $\mathbf{x}$ on a $D$-dimensional lattice of coordination number $b$, at time step $t$. The collision operator

$$
\Omega_{i}^{\alpha}=-\frac{1}{\tau^{\alpha}}\left[n_{i}^{\alpha}(\mathbf{x}, t)-n_{i}^{\alpha e q}(\mathbf{x}, t)\right]
$$

represents the change in the single-particle distribution function due to the collisions. A popular form is the single relaxation time $\tau_{\alpha}$, linear Bhatnagar-Gross-Krook (BGK) form [53] for the collision operator. It can be shown for low Mach numbers that the LB equations correspond to a solution of the Navier-Stokes equation for isothermal, quasiincompressible fluid flow. The lattice Boltzmann method is an excellent candidate to exploit the possibilities of parallel computers, as the dynamics at a lattice site requires only information about quantities at nearest neighbor lattice sites $[24,49]$. The local equilibrium distribution $n_{i}^{\alpha e q}$ plays a fundamental role in the dynamics of the system as shown by Eq. (1). In this study, we use a purely kinetic approach, for which the local equilibrium distribution $n_{i}^{\alpha e q}(\mathbf{x}, t)$ is derived by imposing certain restrictions on the microscopic processes, such as explicit mass and total momentum conservation [54]

$$
n_{i}^{\alpha e q}=\zeta_{i} \rho^{\alpha}\left(1+\frac{\mathbf{c}_{i} \mathbf{u}}{c_{s}^{2}}+\frac{\left(\mathbf{c}_{i} \mathbf{u}\right)^{2}}{2 c_{s}^{4}}-\frac{u^{2}}{2 c_{s}^{2}}+\frac{\left(\mathbf{c}_{i} \mathbf{u}\right)^{3}}{6 c_{s}^{6}}-\frac{u^{2}\left(\mathbf{c}_{i} \mathbf{u}\right)}{2 c_{s}^{4}}\right),
$$

where $\rho^{\alpha}(\mathbf{x}, t) \equiv \Sigma_{i} \eta_{i}^{\alpha}(\mathbf{x}, t)$ is the fluid density and $\mathbf{u}$ $=\mathbf{u}(\mathbf{x}, t)$ is the macroscopic bulk velocity of the fluid, given by $\rho^{\alpha}(\mathbf{x}, t) \mathbf{u}^{\alpha} \equiv \sum_{i} n_{i}^{\alpha}(\mathbf{x}, t) \mathbf{c}_{i}$. $\zeta_{i}$ are the coefficients resulting from the velocity space discretization and $c_{s}$ is the speed of sound, both of which are determined by the choice of the lattice. We use a D3Q19 implementation, i.e., a threedimensional lattice with 19 discrete velocities. Immiscibility of species $\alpha$ is introduced in the model following Shan and Chen $[55,56]$, where only nearest neighbor interactions among the species are considered. These interactions are described by a self-consistently generated mean field body force

$$
\mathbf{F}^{\alpha}(\mathbf{x}, t) \equiv-\psi^{\alpha}(\mathbf{x}, t) \sum_{\bar{\alpha}} g_{\alpha \bar{\alpha}} \sum_{\mathbf{x}^{\prime}} \psi^{\bar{\alpha}}\left(\mathbf{x}^{\prime}, t\right)\left(\mathbf{x}^{\prime}-\mathbf{x}\right),
$$

where $\psi^{\alpha}(\mathbf{x}, t)$ is the so-called effective mass, which can have a general form for modeling various types of fluids [we use $\psi^{\alpha}=\left(1-e^{-\rho^{\alpha}}\right)$ [55]], and $g_{\alpha \bar{\alpha}}$ is a force coupling constant whose magnitude controls the strength of the interaction between components $\alpha$ and $\bar{\alpha}$ and is set positive to mimic repulsion. The dynamical effect of the force is realized in the BGK collision operator by adding to the velocity $\mathbf{u}$ in the equilibrium distribution of Eq. (3) the increment

$$
\delta \mathbf{u}^{\alpha}=\frac{\tau^{\alpha} \mathbf{F}^{\alpha}}{\rho^{\alpha}} .
$$

Amphiphiles are introduced within the model as described in [25] and [43]. An amphiphile usually possesses two different fragments, each having an affinity for one of the two immiscible components. The orientation of any amphiphile present at a lattice site $\mathbf{x}$ is represented by an average dipole vector $\mathbf{d}(\mathbf{x}, t)$. Its direction is allowed to vary continuously and no information is specified for each velocity $\mathbf{c}_{i}$, for reasons of computational efficiency and simplicity. The am- 
phiphile density at a given site is given by an additional fluid species with density $\rho^{s}$. The model has been used successfully to study spinodal decomposition $[2,27]$, the formation of mesophases [5,44-46,48,51,57], and flow in porous media [39].

We use LB3D [49], a highly scalable parallel LB code, to implement the model. The very good scaling of our code permits us to run all our simulations on multiprocessor machines and computational grids in order to reduce the length of data collection to a few weeks. Also, we are able to use simulation boxes typically eight times bigger than previous studies so as to minimize finite size effects.

In order to study the rheological behavior of complex fluid mixtures, we have implemented Lees-Edwards boundary conditions, which were originally developed for molecular dynamics simulations [58]. They reduce finite size effects as compared to moving solid walls [58] and have been used in lattice Boltzmann simulations by various authors $[37,38,40,59]$. This computationally convenient method imposes new positions and velocities on particles leaving the simulation box in the direction perpendicular to the imposed shear strain while leaving the other coordinates unchanged. Choosing $z$ as the direction of shear and $x$ as the direction of the velocity gradient, we have

$$
\begin{gathered}
z^{\prime} \equiv \begin{cases}\left(z+\Delta_{z}\right) \bmod N_{z}, & x>N_{x}, \\
z \bmod N_{z}, & 0 \leq x \leq N_{x}, \\
\left(z-\Delta_{z}\right) \bmod N_{z}, & x<0,\end{cases} \\
u_{z}^{\prime} \equiv \begin{cases}u_{z}+U, & x>N_{x}, \\
u_{z}, & 0 \leq x \leq N_{x}, \\
u_{z}-U, & x<0,\end{cases}
\end{gathered}
$$

where $\Delta_{z} \equiv U \Delta t, U$ is the shear velocity, $u_{z}$ is the $z$ component of $\mathbf{u}$, and $N_{x(z)}$ is the system length in the $x(z)$ direction. We also use an interpolation scheme suggested by Wagner and Pagonabarraga [38] as $\Delta_{z}$ is not generally a multiple of the nearest neighbor lattice distance. For oscillatory shear, we set

$$
U(t)=U \cos (\omega t),
$$

where $\omega / 2 \pi$ is the frequency of oscillation.

In nonsheared studies of spinodal decomposition it has been shown that large lattices are needed to overcome finite size effects. There, $128^{3}$ was the minimum acceptable number of lattice sites [2]. More quantitatively, Kendon et al. [33] set $L / 4$ as the maximum length scale, which is not affected by finite size effects in their spinodal decomposition simulation, where $L$ is the length of the simulation box. We therefore choose $256^{3}$ for all nonsheared simulations to limit the influence of finite size effects even further. For high shear rates, systems also have to be highly extended in the direction of the applied shear because, if the system is too small, the domains interconnect across the $z=0$ and $z=N_{z}$ boundaries to form continuous lamellae in the direction of shear $[39,49]$. Such artifacts need to be eliminated from our simulations. In this case, a good compromise to limit finite size effects and to keep the computational expense as low as pos- sible is a lattice size of $128 \times 128 \times 512$ and this is used here. Mass and relaxation times are always set to unity, i.e., $\tau^{\alpha}$ $=1.0, m^{\alpha}=1.0$. We call the two immiscible fluids "red" and "blue" and set their initial densities to identical values, $\rho^{r}$ $=\rho^{b}$. The initial average surfactant density $\rho^{s}$ is varied between 0.0 and 0.7 . The lattice is then randomly populated with constant initial total fluid densities $\rho^{\text {tot }}=\rho^{r}+\rho^{b}+\rho^{s}$ $=1.6$. This is in contrast to previous studies where only $\rho^{r}$ $+\rho^{b}$ was kept constant [5]. The coupling constant in Eq. (4) between red and blue species is set to $g_{\text {br }}=0.08$, the coupling between an immiscible fluid and surfactant to $g_{\text {bs }}=-0.006$, and the constant describing the strength of the surfactantsurfactant interaction is kept at $g_{\mathrm{ss}}=-0.003$. All units in this paper are given in lattice units if not stated otherwise. While our method has been used to simulate other mesophases like lamellar phases, the primitive $P$ phase [44], and the gyroid phase $[5,46]$, the parameters used in all simulations presented here are known to produce a sponge phase in the absence of bulk flow. More detailed investigations of the particular choice of coupling constants and how they modify the system's properties are given in $[2,5,45,46,51]$.

To analyze the behavior of the various simulations, we define the time-dependent lateral domain size $L(t)$ along direction $i=x, y, z$ as

$$
L_{i}(t) \equiv \frac{2 \pi}{\sqrt{\left\langle k_{i}^{2}(t)\right\rangle}},
$$

where

$$
\left\langle k_{i}^{2}(t)\right\rangle \equiv \frac{\sum_{\mathbf{k}} k_{i}^{2} S(\mathbf{k}, t)}{\sum_{\mathbf{k}} S(\mathbf{k}, t)}
$$

is the second-order moment of the three-dimensional structure function

$$
S(\mathbf{k}, t) \equiv \frac{1}{V}\left|\phi_{\mathbf{k}}^{\prime}(t)\right|^{2}
$$

with respect to the Cartesian component $i,\langle\rangle$ denotes the average in Fourier space, weighted by $S(\mathbf{k}, t)$, and $V$ is the number of nodes of the lattice, $\phi_{\mathbf{k}}^{\prime}(t)$ is the Fourier transform of the fluctuations of the order parameter $\phi^{\prime} \equiv \phi-\langle\phi\rangle$, and $k_{i}$ is the $i$ th component of the wave vector. A projection of the structure function allows us to compare simulation data to scattering patterns obtained in experiments. We obtain those projections by summing up $S(\mathbf{k}, t)$ in one of the Cartesian directions. For example, for the projection in the $z$ direction this leads to $S_{z}\left(k_{x}, k_{y}, t\right)=\Sigma_{k_{z}} S(\mathbf{k}, t)$.

\section{RESULTS}

\section{A. Ternary amphiphilic systems without shear}

Spinodal decomposition of a binary immiscible fluid mixture has been studied extensively within our model by González-Segredo et al. [2]. The authors report domain size scaling as expected for a crossover from diffusive behavior to hydrodynamic viscous growth, i.e., the domain size grows 

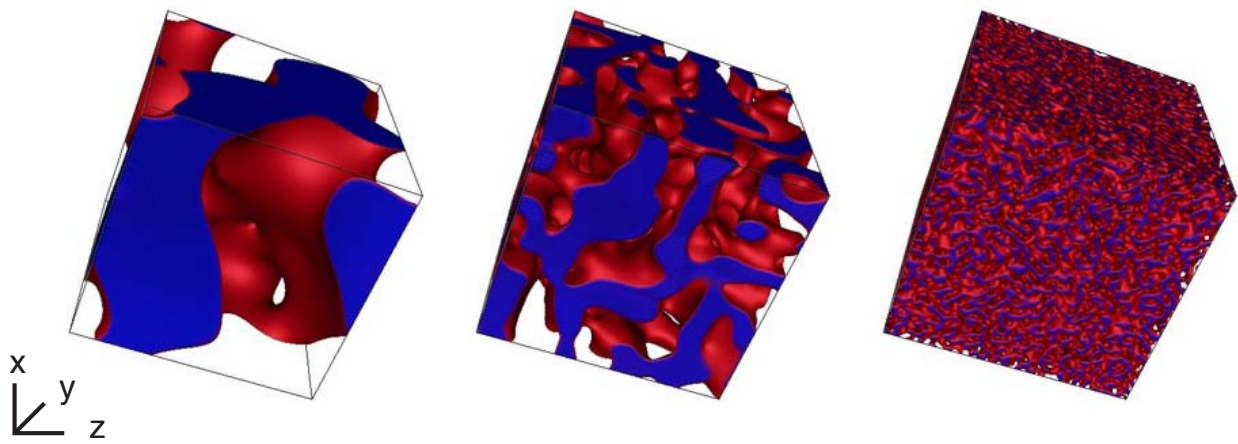

FIG. 1. (Color online) Volume rendered fluid densities of $256^{3}$ systems at $t=30000$ for surfactant densities $\rho^{s}=0.00,0.15,0.30$ (from left to right). For better visibility only one of the immiscible fluid species is shown. Different colors denote the interface and areas of high density of the visualized fluid. The surfactant particles (not shown) are aligned at the interfaces and the second immiscible fluid component fills the void space. After 30000 time steps the phases have separated to a large extent if no surfactant is present (left). Adding a small amount of surfactant $\left(\rho^{s}=0.15\right.$, center) causes the domains to grow more slowly, as depicted by the smaller structures in the volume rendered image. For sufficiently high amphiphile concentrations $\left(\rho^{s}=0.30\right.$, right $)$ the growth process arrests with the formation of a stable bicontinuous microemulsion.

as $L \propto t^{\gamma}$, with $\gamma$ being between $1 / 3$ and 1 . Moreover, they find very good agreement with the dynamical scaling hypothesis, recovering the expected universal behavior of the structure function.

If one adds surfactant to a binary immiscible fluid mixture, the surfactant molecules self-assemble at the interface between the two species and slow down the phase-separation process. For sufficiently high surfactant concentrations, domain growth is arrested completely, leading to a stable microemulsion. In [5], González-Segredo et al. extend their work to ternary amphiphilic fluids and study how the phase separation of a binary immiscible fluid mixture is altered by the presence of surfactant. As already described in the Introduction, by gradually increasing the surfactant concentration they find a slowing down of the domain growth, initially from algebraic to logarithmic temporal dependence, and, at higher surfactant concentrations, from logarithmic to stretched-exponential behavior. They also observe a structural transition from sponge to gyroid phases by increasing the amphiphile concentration or varying the amphiphileamphiphile or amphiphile-binary fluid coupling constants. For growth-arrested mesophases, they observe temporal oscillations of the domain size due to Marangoni flows.

In the present work we use simulation parameters that differ from those of previous studies and are known to produce a sponge phase. In order to avoid effects due to variations of the fluid densities, we also keep the total density in the system constant at 1.6 (in lattice units), while varying the surfactant densities $\rho^{s}$ between 0.00 and 0.70 . Furthermore, our simulation lattices are up to eight times larger in order to keep the influence of finite size effects to a minimum, and we simulate for up to 30000 time steps in order to gain a better understanding of the long time behavior of the system. We study the influence of the amphiphile concentration on the phase-separation process in this section and reproduce previous results with the present parameters. In addition, we study the dependence of the maximum achievable domain size in a stable microemulsion on surfactant concentration as well as the time needed to achieve this state.

To depict the influence of the surfactant density on the phase-separation process, Fig. 1 shows three volume ren- dered $256^{3}$ systems at surfactant densities 0.0 (left), 0.15 (center), and 0.3 (right). As in all figures throughout the paper, for better visibility only one of the immiscible fluid species is shown. Different colors denote the interface and areas of high density of the rendered fluid. The surfactant particles are aligned at the interfaces and the second immiscible constituent fills the void space. After 30000 time steps the phases have separated to a large extent when no surfactant is present (left). Running the simulation for even longer would result in two perfectly separated phases, each of them contained in a single domain only. If one adds some surfactant $\left(\rho^{s}=0.15\right.$, center $)$, the domains grow more slowly, visualized by the smaller structures in the volume rendered image. For sufficiently high amphiphile concentrations $\left(\rho^{s}=0.30\right.$, right $)$ the growth process arrests leading to a stable bicontinuous microemulsion with small individual domains formed by the two immiscible fluids.

The projected structure function $S_{z}\left(k_{x}, k_{y}, t\right)$ ("scattering pattern") is given in Fig. 2 for two surfactant densities $\rho^{s}$ $=0.00$ (a) and 0.30 (b) at time step $t=10000$. As can be clearly seen in Fig. 2(a), a strong peak occurs for small values of $k_{x}, k_{y}$, depicting the occurrence of length scales that are of the order of the system size. For $\rho^{s}=0.30$, however, the peaks are by a factor of 100 smaller and shifted to larger values of $k_{x}, k_{y}$. We find a volcanolike scattering pattern, indicating the dominance of small length scales. Since our system is cubic and no shear is applied, the projections of the structure function in $x$ and $y$ directions are equivalent.

To investigate the influence of surfactant more quantitatively, in Fig. 3 the time-dependent lateral domain size $L(t)$ as given in Eq. (9) is shown for a number of surfactant densities $\rho^{s}$ between 0.00 and 0.50 . Since the lattice is cubic here, $L(t)$ behaves identically in all three directions. Figures 3(a) and 3(b) show identical data, but different scalings of the axes. In Fig. 3(a), we plot the data linearly in order to give a better impression of the time dependence of the growth dynamics. However, in order to check which data are best fitted by the various growth laws, we provide a log-log scale plot of the same data in Fig. 3(b). For the first few hundred time steps, the randomly distributed fluid densities 

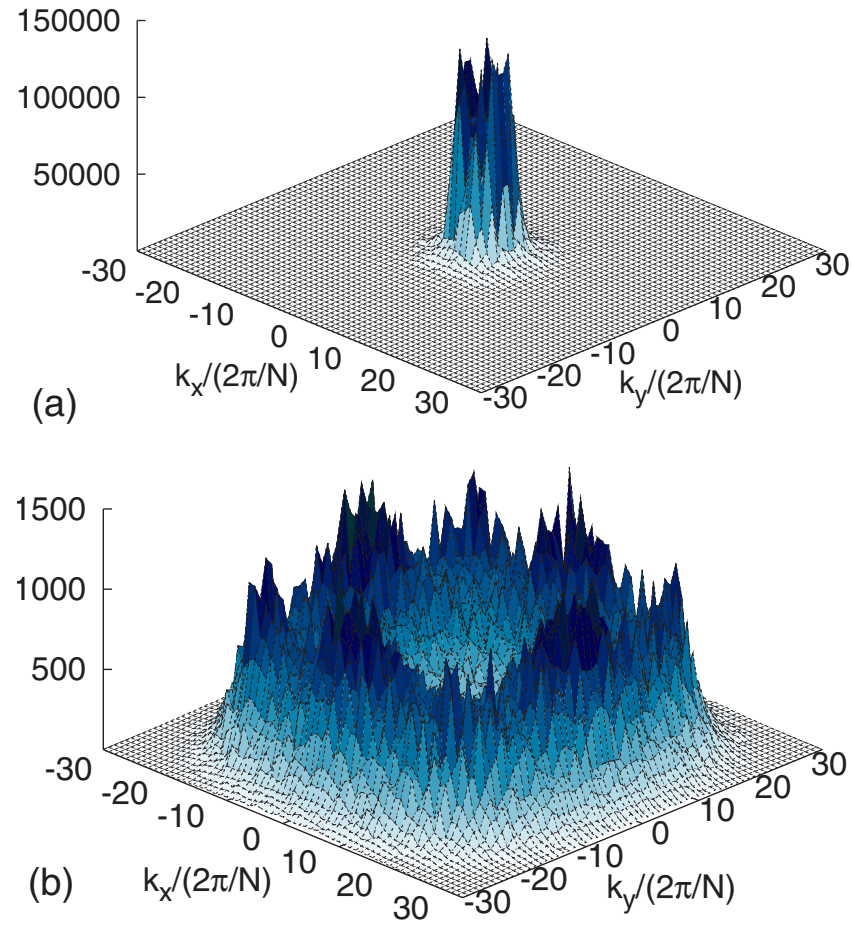

FIG. 2. (Color online) Projected structure function $S_{z}\left(k_{x}, k_{y}, t\right)$ for $\rho^{s}=$ (a) 0.00 and (b) 0.30 at time step $t=10000$. For the case without surfactant, a strong peak occurs for small values of $k_{x}, k_{y}$, reflecting the dominance of length scales that are of the order of the system size. For $\rho^{s}=0.30$, only much smaller peaks occur for larger values of $k_{x}, k_{y}$, indicating that only small length scales are present. All quantities are expressed in lattice units.

of the initial system configuration cause a spontaneous formation of small domains resulting in a steep increase of $L(t)$. For $\rho^{s}=0.00$ domain growth does not come to an end until the domains span the full system. By adding surfactant we

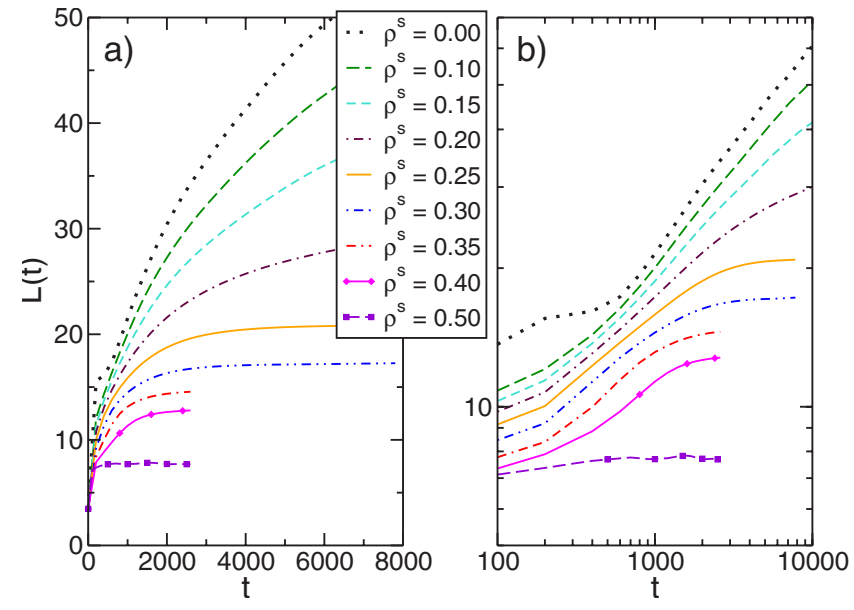

FIG. 3. (Color online) Average domain size $L(t)$ for various surfactant densities $\rho^{s}=0.00,0.10,0.15,0.20,0.25,0.30,0.35,0.40$, 0.50 . Axes are linear in (a) and logarithmic in (b). After the initial spontaneous formation of domains, domain growth can be described by a power law. With increasing $\rho^{s}$ domain growth slows down and eventually comes to an end at a maximum domain size $L_{\max }\left(\rho^{s}\right)$. All quantities are expressed in lattice units.
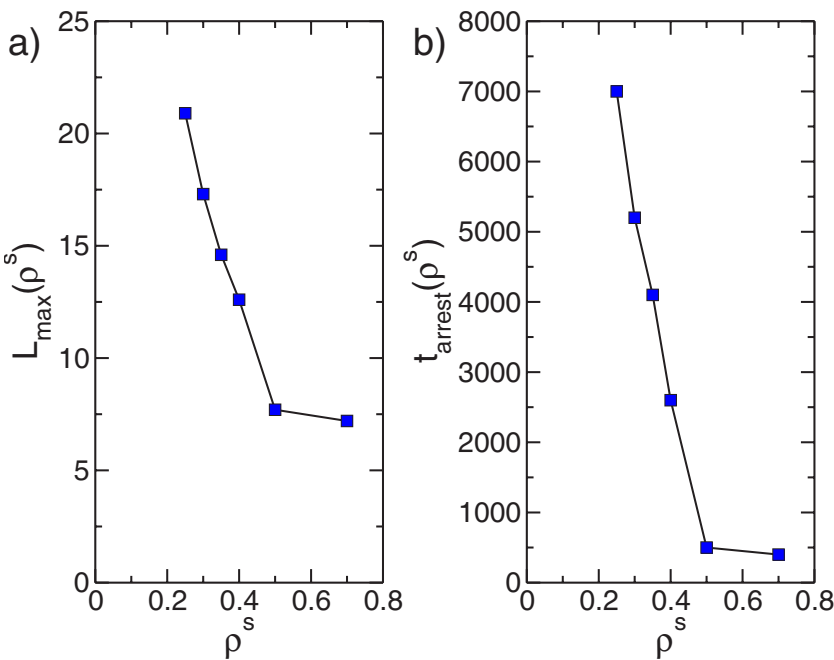

FIG. 4. (Color online) (a) Maximum domain size $L_{\max }\left(\rho^{s}\right)$ and (b) time of arrest $t_{\text {arrest }}\left(\rho^{s}\right)$ for various surfactant densities $\rho^{s}=0.25$, $0.30,0.35,0.40,0.50,0.7 . L_{\max }\left(\rho^{s}\right)$ as well as $t_{\text {arrest }}\left(\rho^{s}\right)$ decrease linearly with increasing surfactant density $\rho^{s}$ and saturate for $\rho^{s}$ $>0.5$. All quantities are expressed in lattice units.

can slow down the growth process, and for high surfactant densities $\rho^{s}>0.25$, the domain growth stops after a few thousand simulation time steps. By adding even more surfactant, the final average domain size becomes very small and does not grow beyond 7.7 lattice sites. We fit our numerical data with the corresponding growth laws and find that for $\rho^{s}$ smaller than $0.15 L(t)$ is best fitted by a function proportional to $t^{\alpha}$. For $\rho^{s}$ being 0.15 or 0.20 , a logarithmic behavior proportional to $(\ln t)^{\theta}$ is observed. Increasing $\rho^{s}$ further results in $L(t)$ being best described by a stretched exponential. These results correspond well with the findings in [5].

We study the dependence of the final domain size $L_{\max }\left(\rho^{s}\right)$ on the amount of surfactant as depicted in Fig. 4(a). It can be observed that the maximum domain size decreases linearly from 20.9 for $\rho^{s}=0.25$ with increasing $\rho^{s}$ until a threshold value is reached at $\rho^{s}=0.5$, where $L_{\max }(t)=7.7$. Then, $L_{\max }\left(\rho^{s}\right)$ decreases much more slowly and stays almost constant. The slope of the linear regime corresponds to -52.8 . The behavior of $L_{\max }\left(\rho^{s}\right)$ and $t_{\text {arrest }}\left(\rho^{s}\right)$ is consistent with previous lattice gas $[4,60]$ and lattice Boltzmann studies [5], where the authors determined the dependence of the surface tension at a planar interface between two immiscible fluid species on the surfactant concentration. In [5], the authors found a linear dependence between surface tension and surfactant density, but they did not study such high concentrations as in the current study. In [60], the surface tension approaches zero for high concentrations, i.e., a saturation occurs, causing the size of the individual fluid domains to saturate as well. These effects can be explained as follows. Adding surfactant to a binary fluid mixture causes the amphiphiles to minimize the free energy in the system by assembling at the interface between the two immiscible fluid species. An increase of surfactant concentration causes the interfacial area to be maximized in order to accommodate as much surfactant as possible. The increasing interfacial area causes the individual domains to become smaller and 

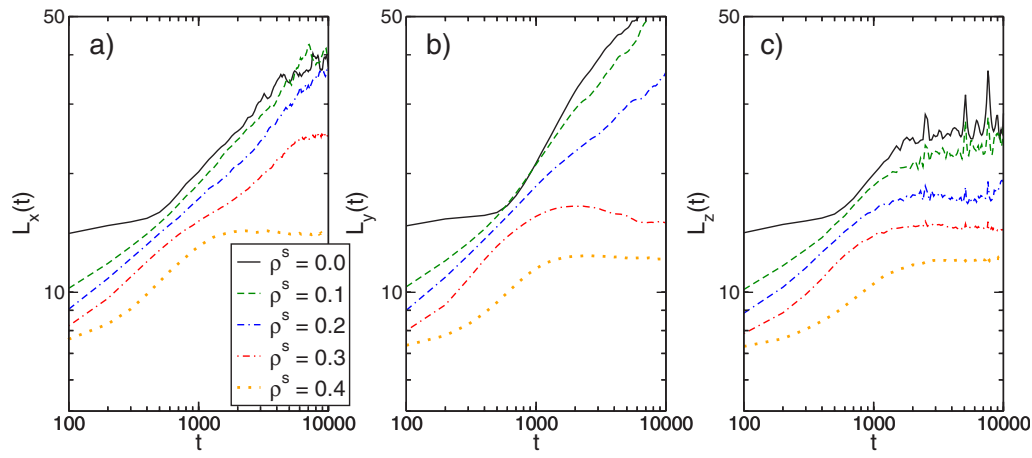

FIG. 5. (Color online) Domain size $L\left(\rho^{s}\right)$ in $x(\mathrm{a}), y(\mathrm{~b})$, and $z$ directions (c) for surfactant densities $\rho^{s}=0.0,0.1,0.2,0.3,0.4$ and a constant shear rate $\dot{\gamma}=1.56$ $\times 10^{-3}$. All quantities are reported in lattice units.

$L_{\max }\left(\rho^{s}\right)$ decreases. If the surfactant concentration becomes very high $\left(\rho^{s}>0.5\right.$ in our case), $L_{\max }\left(\rho^{s}\right)$ saturates due to the maximum possible interfacial area being reached and all available area being covered with surfactant molecules. More amphiphiles accumulating at the interface would lead to very steep and energetically unfavorable gradients of surfactant density in the system. Therefore, further amphiphiles have to reside within the bulk fluid phases, forming micellar structures. Within our model the minimum final domain size corresponds to 7.7 lattice sites. However, this value can be varied by tuning the coupling constants for the amphiphileamphiphile or amphiphile-fluid interactions. A more thorough investigation of the influence of the particular choice of the coupling constants on the final domain size is of particular interest and will be investigated within a future project.

In Fig. 4(b), the number of simulation time steps $t_{\text {arrest }}\left(\rho^{s}\right)$ needed to reach the final domain size is plotted. Since the time it takes for the system to relax to its equilibrium state directly depends on the final domain size, it is consistent with the data presented in Fig. 4(a) that a linear dependence of $t_{\text {arrest }}\left(\rho^{s}\right)$ on the surfactant concentration can be observed. While for $\rho^{s}=0.257000$ time steps are needed to reach the maximum possible domain size, for $\rho^{s}=0.5500$ time steps are sufficient. For $\rho^{s}>0.5, t_{\text {arrest }}\left(\rho^{s}\right)$ decreases much more slowly than for $\rho^{s}<0.5$. The slope of $t_{\text {arrest }}\left(\rho^{s}\right)$ in the linear regime is given by -26000 .

\section{B. Steadily sheared systems}

If a binary immiscible fluid mixture is driven mechanically by external shear forces, it is known that the evolution of domains and phase-separation processes is changed profoundly [8-10]. The most noticeable effect is the formation of a lamellar phase, i.e., elongated domains or lamellae form and align along the flow direction. Due to the anisotropy of the system, the time-dependent domain size $L(t)$ behaves differently for the different coordinate axes in this case. Furthermore, modified growth exponents are expected due to the anisotropic effects.

As already seen in the previous section, adding amphiphiles to a binary immiscible fluid under shear can change its properties dramatically. The amphiphiles stabilize the interface between the immiscible fluid species and the domain growth is hindered as described in the previous section. Moreover, the amphiphiles might form complex structures which can have an impact on the properties of the sheared fluid leading to non-Newtonian flow $[8,45,51]$.

We study ternary $128 \times 128 \times 512$ sized systems under constant shear. The shear rate is set to $\dot{\gamma}=1.56 \times 10^{-3}$ and $3.12 \times 10^{-3}$, while the surfactant density is varied between $\rho^{s}=0.0$ and 0.4 . Figure 5 shows the time-dependent lateral domain size for all three coordinate axes at $\dot{\gamma}=1.56 \times 10^{-3}$. In the $x$ direction, which is the axis between the shear planes, the power law regime of $L_{x}(t)$ starts at $t=500$ for the $\rho^{s}$ $=0.0$ case, while for higher $\rho^{s}$ the initial growth regime is overcome by the power law regime before the first measurement at $t=100$. As long as $\rho^{s}<0.3$, the growth rate is not hindered by the amphiphiles and domains grow until the end of the simulation. For $\rho^{s}=0.3$ the power law regime starts to breakdown at $t=900$ and $L_{x}(t)$ saturates at $t=5000$. Adding even more surfactant results in an even earlier saturation at $t=1500$. The $y$ direction is the direction parallel to the shear planes and perpendicular to the direction of shear. Since this direction is less affected by the shear forces, $L_{y}(t)$ grows faster than $L_{x}(t)$ for low surfactant concentrations $\rho^{s}<0.2$, causing the domains forming to be extended in the $y$ direction. For $\rho^{s}=0.2 L_{x}(t)$ and $L_{y}(t)$ behave almost identically, while for $\rho^{s}>0.2$ a crossover occurs and the maximum attainable value for $L_{y}(t)$ is below the result for $L_{x}(t)$. In the direction of shear ( $z$ direction), $L_{z}(t)$ saturates even for the no surfactant case at $L_{z}(t)=25$ and comes to arrest at even smaller values with increasing $\rho^{s}$. The complex behavior of $L_{i}(t)$ can be better understood by reminding ourselves that the domain size is measured in the direction of the Cartesian coordinate axes. However, individual fluid domains occurring in the system are being elongated due to the shear and try to align with the shear gradient. Thus, they are not parallel to any coordinate axis. Therefore, with a measurement of $L_{z}(t)$ one is not able to detect the actual length of individual lamellae, but only their thickness in the $z$ direction. Similar arguments are valid for $L_{x}(t)$, shear causing the measured domain size in the $x$ direction to be larger than the lamellae's thickness. For increasing $\rho^{s}$, the average domain size reduces due to the influence of the amphiphiles, thus causing the individual domains to become smaller. If $\rho^{s}$ $>0.2$, the alignment of the domains with the shear causes $L_{x}(t)$ to appear larger than $L_{z}(t)$. For high surfactant concentrations $\left(\rho^{s}=0.4\right)$ all three directions behave very similarly: domain growth comes to an end after fewer than 2000 time steps and the final domain size is between 10 and 15 lattice 


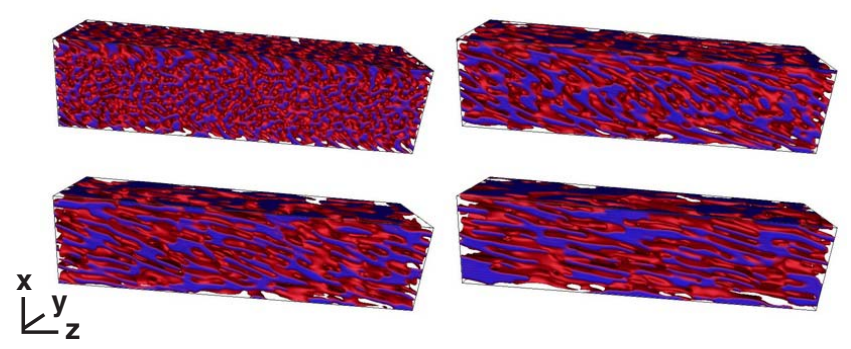

FIG. 6. (Color online) Volume rendered $(128 \times 128 \times 512$ simulation boxes) fluid densities for surfactant density $\rho^{s}=0.2$, a constant shear rate $\dot{\gamma}=1.56 \times 10^{-3}$, and variable number of time steps $t=1000$ (upper left), 4000 (upper right), 6000 (lower left), and 10000 (lower right). While the shape of individual domains does not show distinct features at early times of the simulation, elongated structures appear at $t=4000$ and start to become aligned with the shear at $t=6000$. At late stages of the simulation run $(t=10000)$, the lamellar phase characterized by thin and long lamellae filling the whole system can be observed.

units in all directions, signaling the appearance of a stable microemulsion where the shape of the domains is almost unaffected by the shear.

Regular peaks occur in $L_{z}(t)$ at every 2500 time steps with less pronounced peaks in between them. These peaks can be explained as follows. For the stretching of domains, a certain amount of work against surface tension is needed. On macroscopic scales, the stress tensor does not vanish due to the viscoelastic response of the system $[61,62]$. On the microscale, however, a breakup and recombination of domains can be observed [63]. These domains grow by diffusion and eventually join each other to form larger structures. If the internal stress becomes too large due to the shear-induced deformation, they break up and start to form again. Assuming a large system with many independent domains growing and breaking incoherently, the only observable effect might be a slowing down of the domain growth. In contrast, if the growth and breakup occur coherently as they do in our simulations, a periodicity in the measured time-dependent domain size can be observed [64]. As can be observed in Fig. 5(c), the frequency of domain breakup is independent of the surfactant concentration, while the height of the peaks decreases with increasing $\rho^{s}$.

Figure 6 shows volume rendered examples of a simulated system with surfactant density $\rho^{s}=0.2$ and a constant shear rate of $\dot{\gamma}=1.56 \times 10^{-3}$. The four snapshots are taken a different times $t=1000$ (upper left), 4000 (upper right), 6000 (lower left), and 10000 (lower right). It can be observed that, at early stages of the simulation, the shape of individual domains does not show distinct features, while at $t=4000$, slightly elongated domains start to occur which begin to align with the shear gradient. At $t=6000$, these features are substantially more dominant and at late simulation times $(t$ $=10000)$ the system is filled with elongated and thin lamellae consisting of one of the immiscible fluid species and which are almost parallel to the shear plane.

In order to permit a comparison with experimentally available scattering data, in Fig. 7 we present projected structure functions for the surfactantless case [Figs. 7(a)-7(c)] and a surfactant density of $\rho^{s}=0.30$ [Figs. 7(d)-7(f)] at $t$ $=10000$. The $x, y$, and $z$ directions are shown from top to bottom. The shown projections are for a cubic $128^{3}$ cutout of the elongated systems. In contrast to the nonsheared case, all three directions show distinct properties. For $\rho^{s}=0.00$, a high peak of $S_{z}\left(k_{x}, k_{y}, t\right)$ can be observed around $k_{x}=k_{y}=0$, while in the $x$ and $y$ directions two lower peaks at positions above zero show up. These data can be interpreted as follows. At $t=10000$, the domain size in the direction of the flow corresponds to the size of the cubic cutout, i.e., 128 lattice sites. In the $x$ and $y$ directions, however, the size of the occurring structures is smaller, indicating the occurrence of very long lamellar structures in the system. By adding surfactant to the system [Figs. 7(d)-7(f)], the occurring length scales depicted decrease by the splitting of the single peak in the $z$ projection and the occurrence of two small peaks at $k_{x}=0$ and $k_{y}$ $= \pm 10$. While the peaks in the $y$ direction denote similar length scales as in the $z$ direction, the projections in the direction between the shear planes $(x)$ show a different behavior. Here, two parallel structures at $k_{y}= \pm 10$ and $k_{z}$ between -20 and 20 indicate a much wider variation of the thickness of the individual domains. This is in contrast to the nonsheared case in Fig. 2, where a volcanolike shape of the structure factor was observed.

Doubling the shear rate to $\dot{\gamma}=3.12 \times 10^{-3}$ results in very similar behavior, as shown in Fig. 8. In the $z$ direction, peaks can now be observed even for $\rho^{s}=0.4$, but $L_{z}(t)$ is much noisier for lower surfactant concentrations. However, it can still be seen that there is a number of equidistant peaks for $\rho^{s}<0.4$ which occur every 2500 time steps with some additional peaks in between in the case of $\rho^{s}=0.0$ and 0.1 . The equidistant peaks occurring with the same frequency as in the $\dot{\gamma}=1.56 \times 10^{-3}$ case shows that the breakup phenomena observed are independent of the shear rate.

A number of experiments have reported that the shear stabilizes the system and causes the phase separation to come to an end with $L_{z}(t)$ being very large and $L_{x}(t)$ being much smaller $[30,40,65,66]$. We are not able to reproduce these results due to the limited size of our simulations: substantially larger systems and higher shear rates need to be studied in order to quantify the arrest of domain growth due to shear. However, the computational resources needed would be at the limit of what can be done on current supercomputers.

We have shown in this section that the dynamical scaling hypothesis does not hold for sheared ternary systems in three dimensions since we indeed find three individual length scales pointing out the transition from the sponge to the lamellar phase: while in the flow direction $(z), L(t)$ is determined by the resultant length of the occurring lamellae, in the direction between the shear planes $(x)$, the domains grow steadily and exhibit power law behavior up to a maximum that depends on the surfactant concentration. In the $y$ direction, domain growth is not hindered by shear. In fact, $L_{y}(t)$ grows slightly faster than in the nonsheared case. Increasing the surfactant concentration has a strong impact on domain growth: starting at $\rho^{s}=0.3, L_{y}(t)$ and $L_{z}(t)$ recover the behav- 


$$
\rho^{\mathbf{s}}=\mathbf{0 . 0}
$$

a)

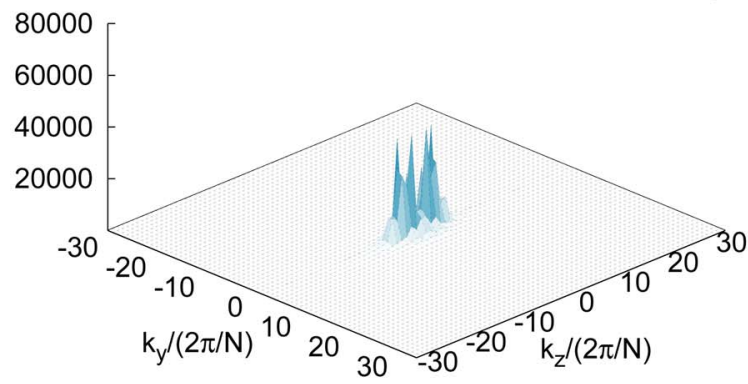

b)

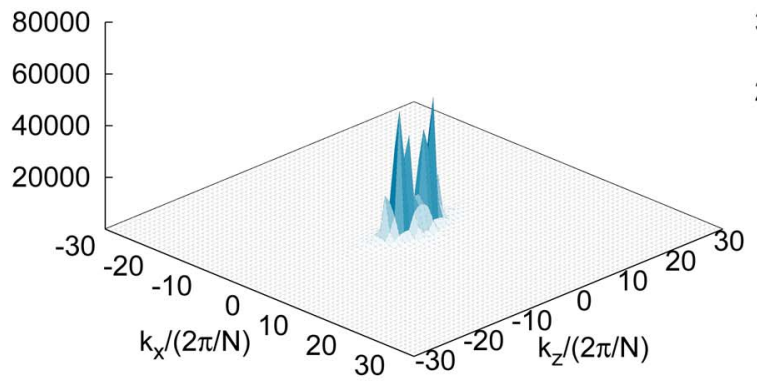

c)

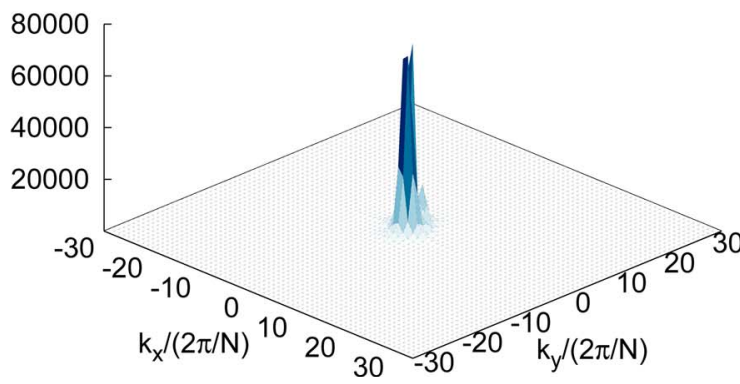

$\rho^{\mathrm{s}}=\mathbf{0 . 3}$

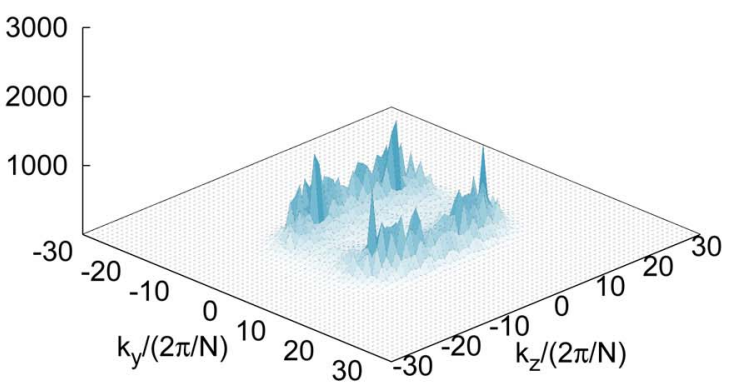

e)

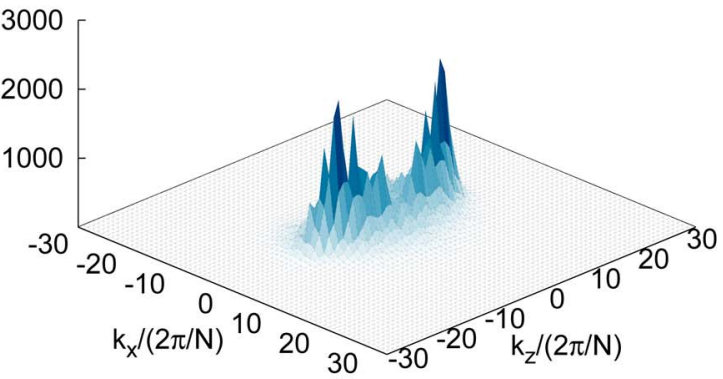

f)

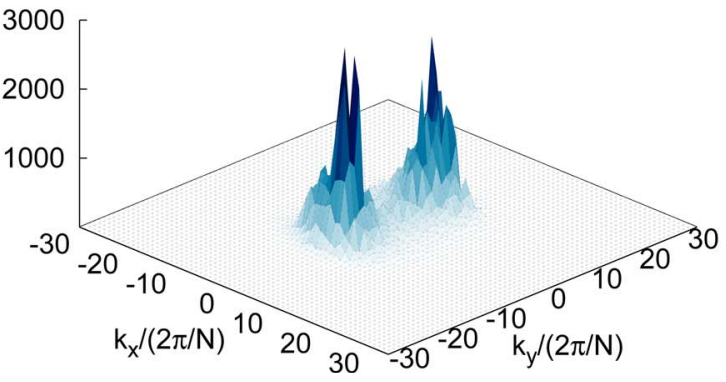

FIG. 7. (Color online) Projected structure functions (scattering pattern) $S_{i}$ for surfactant concentrations $\rho^{s}=0.0$ (a) - (c) and 0.3 (d) - (f) at $t=10$ 000. Projections are in the $x, y$, and $z$ directions (from top to bottom) and for a cubic cutout of the full system with side length $N$ $=128$.

ior of the case without shear, i.e., the length scales saturate around 15. In the $x$ direction, however, growth continues up to $L_{x}(t)=26$. This can be explained as follows. With increasing surfactant concentration, the final domain sizes become smaller, reducing the influence of the shear forces in the $y$ and $z$ directions. In the direction between the shear planes, however, an increase of $L_{x}(t)$ can still be observed because the domains are still being elongated due to shear and try to align with the velocity profile. Thus, they are tilted and their size appears to be smaller than it actually is in the $z$ direction and larger in the $x$ direction.

Our findings are in agreement with Ginzburg-Landau and Langevin calculations [64,67-69] as well as twodimensional lattice Boltzmann simulations of binary immiscible fluid mixtures as presented in $[31,36,40]$. However, to the best of our knowledge, there are no detailed theoretical studies of the dependence of domain growth properties on the surfactant concentration. The only known work utilizes a Ginzburg-Landau free-energy approach to study sheared mi- croemulsions, but does not vary the amount of surfactant. In addition, the authors only cover two-dimensional systems and are thus unable to describe the behavior of $L_{y}(t)$ [70].

\section{Complex fluids under oscillatory shear}

In the case of oscillatory shear, the morphology and the domain growth are altered significantly, although the average deformation is zero after each period of shear. For example, it has been found experimentally for binary fluid mixtures that for very low oscillation frequencies domain growth can be interrupted [71], or domains can grow on much longer time scales than given by the oscillation frequency [72]. Simulations so far either do not include hydrodynamic effects, or are limited to two dimensions [14]. It has been observed in the two-dimensional lattice Boltzmann studies by $\mathrm{Xu}$ et al. that hydrodynamic effects must not be neglected in the case of oscillatory shear since there exists a finite time inversely proportional to the viscosity which is required to 

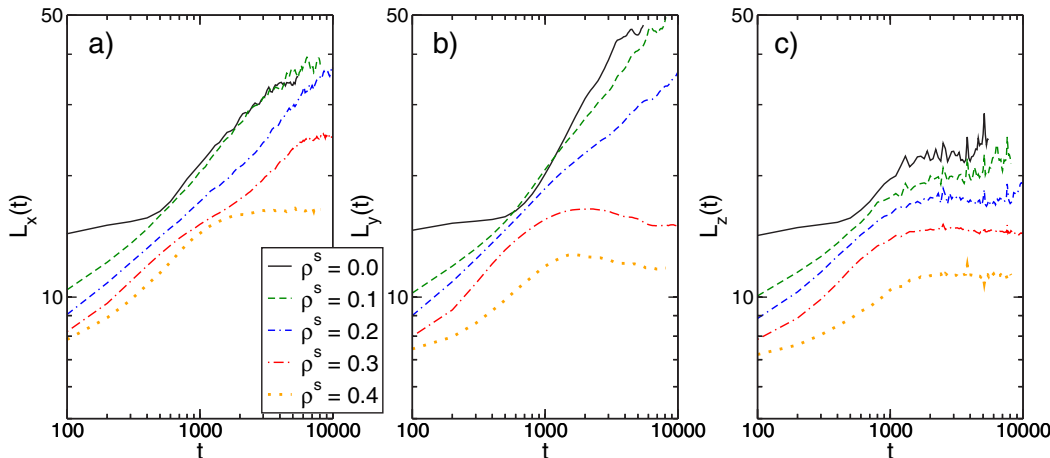

FIG. 8. (Color online) Domain size $L\left(\rho^{s}\right)$ in (a) $x$, (b) $y$, and (c) $z$ directions for surfactant densities $\rho^{s}=, 0.1,0.2,0.3,0.4$ and a constant shear rate $\dot{\gamma}=3.12 \times 10^{-3}$. set a linear velocity profile in the system. For high oscillation frequencies, this time is longer than the oscillation period and there will never be a linear velocity profile in the system, thus influencing the domain growth substantially [14].

Due to its higher complexity, oscillatory shear has been a subject of much less research than systems under steady shear. Therefore, the number of publications that can be found in the literature is substantially smaller. To the best of our knowledge, no detailed three-dimensional simulation studies of phase separation in ternary amphiphilic fluid mixtures under oscillatory shear have been reported so far. The most detailed three-dimensional simulations we are aware of are our own studies of the gyroid mesophase under oscillatory shear as presented in [45].

In this section we present our results of simulations of systems equivalent to the ones considered in the previous section, but with the shear plane moved as given by Eq. (8). We apply two different oscillation frequencies $\omega=0.001$ and 0.01 , where a single oscillation takes 6283 time steps in the slow case and 628.3 time steps in the fast case.

Let us first consider the case with a lower oscillation frequency and lower shear rate, i.e., $\omega=0.001$ and $\dot{\gamma}=1.56$ $\times 10^{-3}$. In the case of oscillatory sheared systems, the individual fluid domains try to align with the velocity gradient as in the previous section. However, since we do not consider steadily moving shear planes here, domains are never able to reach a steady state and instead have to follow the oscillation of the planes. The frequencies considered in our simulations are comparably high since no linear velocity gradient sustains long enough during a single oscillation for the domains to fully align with it. This is depicted in Fig. 9 which shows two typical examples from a simulation with $\rho^{s}=0.2$. On the left hand side, a volume rendered snapshot is given at $t$ $=2500$. Here, the oscillating shear planes have just passed their reversal point. Close to the shear planes, the domains are aligned vertically because they have to be turned around in order to follow the changing direction of movement of the shear planes. In the bulk of the system, however, no preferred direction can be observed since the velocity gradient does not interpenetrate the whole system. At $t=10000$, the shear planes are in a position just before their reversal point. Thus, the fluid mixture was accelerated for more than 2000 time steps and the domains close to the shear boundary are well aligned in the direction of the flow. In the bulk, again no preferred direction can be observed.

The time-dependent lateral domain size of this simulation and for varied surfactant concentration is presented in Fig.
10. As in the case of continuous shear, the domain growth in $y$ direction is almost uninfluenced by the applied shear forces. In fact, for low surfactant concentrations $\rho^{s}<0.2$, $L_{y}(t)$ even grows slightly faster than in the case without oscillatory movement. For $\rho^{s} \geq 0.2$ the maximum domain size obtained is similar to the steady shear case. Due to the nonsteady movement of the shear planes, $L_{x}(t)$ and $L_{z}(t)$ show a richer behavior: both functions show distinct kinks around the reversal points of the shear and for $\rho^{s} \geq 0.2$ it is found that the growth rates are smaller than in the case of steady shear. Thus, we can observe the formation of tubular structures which are elongated in the $y$ direction and show similar length scales in the $x$ and $z$ directions. For very high surfactant concentrations $\left(\rho^{s}=0.4\right)$ it is not possible to distinguish between tubular and spherical structures due to the small size of the individual domains.

In the $z$ direction we can still observe peaks related to the formation and breakup, as well as the rotational movement, of domains. However, due to the overlaid effect of the oscillation, these peaks are no longer equidistant as in the steady shear case.

In Fig. 11 we present a system with a ten times larger oscillation frequency, i.e., $\omega=0.01$. Here, the frequency of the oscillations is so high that the fluid is no longer able to follow the movement of the walls. Thus, the influence of the shear on the growth behavior becomes less pronounced, with the domains constantly growing as long as the amount of surfactant present allows it. The growth rates are comparable to those in the nonsheared case here and show identical growth laws as in the nonsheared case. The only difference is

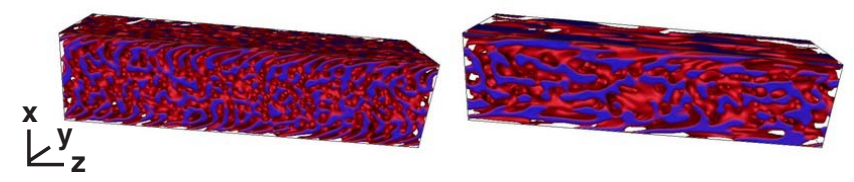

FIG. 9. (Color online) Volume rendered fluid densities for surfactant density $\rho^{s}=0.2$ at $t=2500$ (left) and 10000 (right). The shear rate is $\dot{\gamma}=1.56 \times 10^{-3}$ and $\omega=0.001$. At $t=2500$, the shear velocity is close to its reversal point and the domains are aligned vertically close to the shear plane, while in the bulk of the system no preferred orientation can be observed. After 10000 time steps, however, the domains close to the shear plane are aligned with the flow direction because the shear planes are in a position just before their reversal point. In the bulk, still no preferred orientation can be found since it takes longer for the velocity gradient to penetrate the whole system than the duration of a single period of shear. 

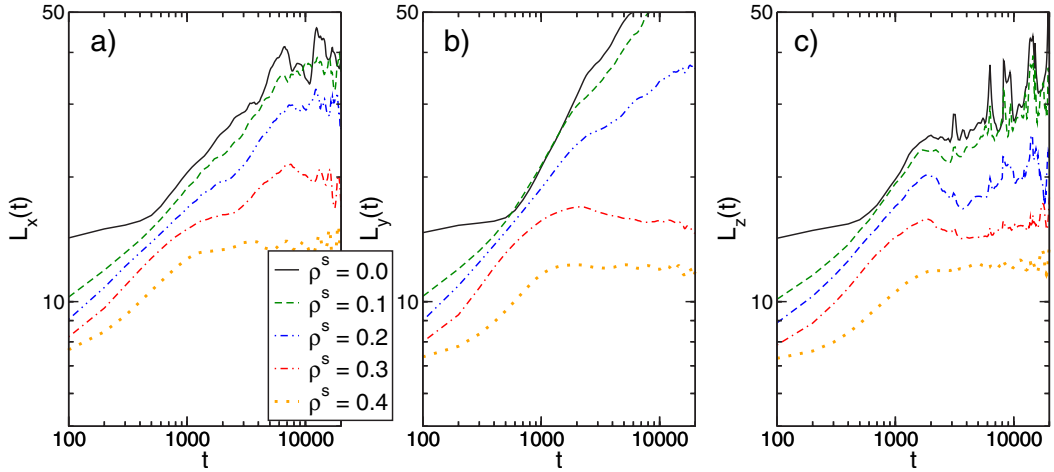

FIG. 10. (Color online) Domain size $L\left(\rho^{s}\right)$ in $x(\mathrm{a}), y(\mathrm{~b})$, and $z$ directions (c) for surfactant densities $\rho^{s}=0,0.1,0.2,0.3,0.4$ and oscillatory shear with $\dot{\gamma}=1.56$ $\times 10^{-3}, \omega=0.001$. that the exponents are found to be smaller while $L_{y}(t)$ grows slightly faster than $L_{x}(t)$ and $L_{z}(t)$, depicting the occurrence of tubular structures in the system. The $z$ direction is the only component of the time-dependent lateral domain size that differs from the unsheared case, because strong oscillations start to appear due to the distortions caused by the moving boundaries.

We have increased the shear rate to $\dot{\gamma}=3.12 \times 10^{-3}$, but the lateral domain sizes obtained are almost identical to those in the $\dot{\gamma}=1.56 \times 10^{-3}$ case. Therefore, we do not present an additional figure, but our findings can be used to argue that for high oscillation frequencies the system behaves in a manner equivalent to the nonsheared case.

In the case of oscillatory shear we have shown the occurrence of tubular structures due to shear-imposed anisotropic domain growth, the slowing down of the domain growth rate depending on the oscillation frequency, as well as the result that a microemulsion with high surfactant concentration stays unaffected by external shear forces. Our results are in agreement with the simulations of Qiu et al. [11] and the two-dimensional lattice Boltzmann simulations of Xu et al. [14].

\section{CONCLUSIONS}

In this paper we have presented detailed threedimensional lattice Boltzmann studies of binary immiscible and ternary amphiphilic fluid mixtures under constant and oscillatory shear.

We have reproduced the well-known power law growth of domains in the case of binary immiscible fluids (spinodal decomposition) which crosses over to a logarithmic law and to a stretched exponential if one increases the surfactant con- centration even further. For sufficiently high surfactant concentrations, domain growth can come to an end and the system corresponds to a stable bicontinuous microemulsion. For amphiphile concentrations of up to $30 \%$ we find linear dependencies of the time of arrest as well as the maximum domain size on the amphiphile concentration. For concentrations above 30\%, neither arrest length nor time of arrest changes any more since the surface tension at the interfaces between the two immiscible fluids is at its minimum. The whole interface is filled with amphiphiles and further amphiphile molecules have to reside within the bulk fluid.

In sheared systems, we have studied the influence of moving boundaries on the effect of domain growth and report domain breakup phenomena depending on the shear rate as well as the amphiphile concentration. Depending on the surfactant concentration and the shear rate, we find a transition from a sponge phase to a lamellar phase.

Under oscillatory shear and with the oscillation frequencies chosen, no linear velocity gradient can build up within a single period of shear. Thus, the domains are constantly rearranging and align with the flow in the vicinity of the shear planes. In the bulk, however, no preferred alignment can be observed. But since the growth in the $y$ direction is not hindered by the shear, tubular structures occur and are best observable for low surfactant concentrations. For very fast oscillations $(\omega=0.01)$, the system is not able to follow the external shear at all. Thus, it behaves similarly to a nonsheared one. The only differences are that fluctuations in $L_{z}(t)$ can be observed due to the oscillatory forces, while the growth in the other two directions is slowed down. For surfactant concentrations $\rho^{s} \leq 0.2$ anisotropic growth in the $x$ and $y$ directions is observed depicting the presence of tubular domains in the system. In future work it would be of interest to study the formation of tubes and their dependency on the
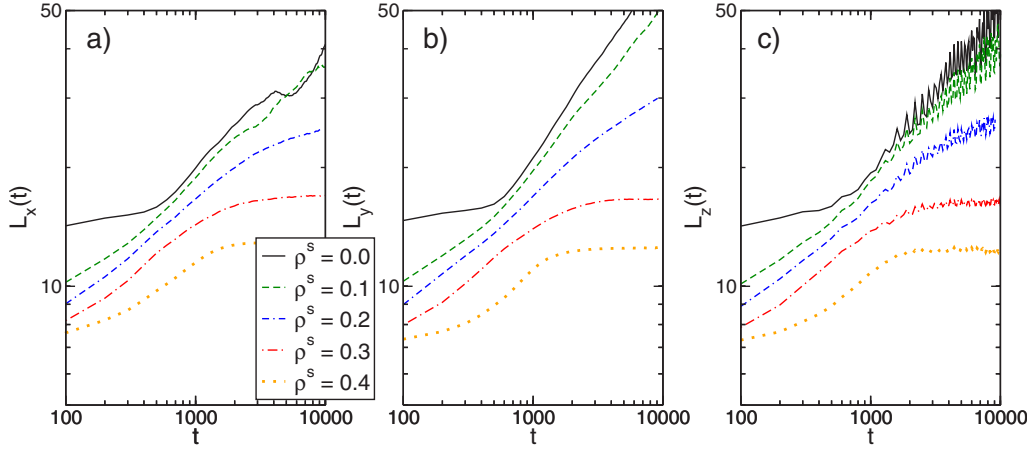

FIG. 11. (Color online) Domain size $L\left(\rho^{s}\right)$ in $x(\mathrm{a}), y(\mathrm{~b})$, and $z$ directions (c) for surfactant densities $\rho^{s}=0,0.1,0.2,0.3,0.4$ and oscillatory shear with $\dot{\gamma}=1.56$ $\times 10^{-3}, \omega=0.01$. 
shear rate, oscillation frequency, and surfactant concentration in greater detail. Additionally, the study of asymmetric mixtures, where the concentrations of the two immiscible fluid species differs, remains unexplored.

\section{ACKNOWLEDGMENTS}

We are grateful for the support of the HPC-Europa program, funded under the European Commission's Research
Infrastructures activity, Contract No. RII3-CT-2003-506079. We also acknowledge RealityGrid and ESLEA EPSRC Grant Nos. GR/R67699 and GR/T04465, the Höchstleistungsrechenzentrum Stuttgart for providing access to their NEC SX8, and the National Science Foundation (NSF) for U.S. TeraGrid usage within NRAC Grant No. MCA04N014. We would like to thank H. Berger, R. Keller, and P. Lammers for technical support and K. Stratford for fruitful discussions.
[1] A. J. Bray, Adv. Phys. 43, 357 (1994).

[2] N. González-Segredo, M. Nekovee, and P. V. Coveney, Phys. Rev. E 67, 046304 (2003).

[3] T. Kawakatsu, K. Kawasaki, M. Furusaka, H. Obayashi, and T. Kanaya, J. Comput. Phys. 99, 8200 (1993).

[4] A. N. Emerton, P. V. Coveney, and B. M. Boghosian, Phys. Rev. E 56, 1286 (1997).

[5] N. González-Segredo and P. V. Coveney, Phys. Rev. E 69, 061501 (2004).

[6] R. A. L. Jones, Soft Condensed Matter (Oxford University Press, Oxford, 2003).

[7] G. Gompper and M. Schick, in Phase Transitions and Critical Phenomena, edited by C. Domb and J. Lebowitz (Academic Press, New York, 1994), Vol. 16, pp. 1-176.

[8] C. Meyer, S. Asnacios, C. Bourgaux, and M. Kleman, Rheol. Acta 39, 223 (2000).

[9] J. Zipfel, J. Berghausen, G. Schmidt, P. Lindner, M. Tsianou, P. Alexandridis, and W. Richtering, Phys. Chem. Chem. Phys. 1, 3905 (1999).

[10] J. Berghausen, J. Zipfel, O. Diat, T. Narayanan, and W. Richtering, Phys. Chem. Chem. Phys. 2, 3623 (2000).

[11] F. Qiu, H. Zhang, and Y. Yang, J. Comput. Phys. 109, 1575 (1998).

[12] Y. Zhang, U. Wiesner, Y. Yang, T. Pakula, and H. Spiess, Macromolecules 29, 5427 (1996).

[13] Y. Zhang and U. Wiesner, J. Comput. Phys. 103, 4784 (1995).

[14] A. Xu, G. Gonnella, and A. Lamura, Phys. Rev. E 67, 056105 (2003).

[15] P. Español and P. Warren, Europhys. Lett. 30, 191 (1995).

[16] S. Jury, P. Bladon, M. Cates, S. Krishna, M. Hagen, N. Ruddock, and P. Warren, Phys. Chem. Chem. Phys. 1, 2051 (1999).

[17] E. G. Flekkøy, P. V. Coveney, and G. De Fabritiis, Phys. Rev. E 62, 2140 (2000).

[18] J.-P. Rivet and J. P. Boon, Lattice Gas Hydrodynamics (Cambridge University Press, Cambridge, U.K., 2001).

[19] A. Malevanets and R. Kapral, Europhys. Lett. 44, 552 (1998).

[20] Y. Hashimoto, Y. Chen, and H. Ohashi, Comput. Phys. Commun. 129, 56 (2000).

[21] T. Sakai, Y. Chen, and H. Ohashi, Comput. Phys. Commun. 129, 75 (2000).

[22] S. Succi, The Lattice Boltzmann Equation for Fluid Dynamics and Beyond (Oxford University Press, Oxford, 2001).

[23] R. Benzi, S. Succi, and M. Vergassola, Phys. Rep. 222, 145 (1992).

[24] P. J. Love, M. Nekovee, P. V. Coveney, J. Chin, N. González-
Segredo, and J. M. R. Martin, Comput. Phys. Commun. 153, 340 (2003).

[25] H. Chen, B. M. Boghosian, P. V. Coveney, and M. Nekovee, Proc. R. Soc. London, Ser. A 456, 2043 (2000).

[26] W. R. Osborn, E. Orlandini, M. R. Swift, J. M. Yeomans, and J. R. Banavar, Phys. Rev. Lett. 75, 4031 (1995).

[27] J. Chin and P. V. Coveney, Phys. Rev. E 66, 016303 (2002).

[28] J. Chin, E. S. Boek, and P. V. Coveney, Proc. R. Soc. London, Ser. A 360, 547 (2002).

[29] F. J. Alexander, S. Chen, and D. W. Grunau, Phys. Rev. B 48, 634 (1993).

[30] A. J. Wagner and J. M. Yeomans, Phys. Rev. Lett. 80, 1429 (1998).

[31] G. Gonnella, E. Orlandini, and J. M. Yeomans, Phys. Rev. Lett. 78, 1695 (1997).

[32] M. E. Cates, V. M. Kendon, P. Bladon, and J. C. Desplat, Faraday Discuss. 112, 1 (1999).

[33] V. M. Kendon, J. C. Desplat, P. Bladon, and M. E. Cates, Phys. Rev. Lett. 83, 576 (1999).

[34] V. M. Kendon, M. E. Cates, I. Pagonabarraga, J. C. Desplat, and P. Bladon, J. Fluid Mech. 440, 147 (2001).

[35] I. Pagonabarraga, J. C. Desplat, A. J. Wagner, and M. E. Cates, New J. Phys. 3, 9.1 (2001).

[36] A. Xu, G. Gonnella, and A. Lamura, Physica A 331, 10 (2004).

[37] A. J. Wagner and J. M. Yeomans, Phys. Rev. E 59, 4366 (1999).

[38] A. Wagner and I. Pagonabarraga, J. Stat. Phys. 107, 521 (2002).

[39] J. Harting, M. Venturoli, and P. V. Coveney, Philos. Trans. R. Soc. London, Ser. A 362, 1703 (2004).

[40] P. Stansell, K. Stratford, J. C. Desplat, R. Adhikari, and M. E. Cates, Phys. Rev. Lett. 96, 085701 (2006).

[41] O. Theissen, G. Gompper, and D. M. Kroll, Europhys. Lett. 42, 419 (1998).

[42] A. Lamura, G. Gonnella, and J. M. Yeomans, Europhys. Lett. 45, 314 (1999).

[43] M. Nekovee, P. V. Coveney, H. Chen, and B. M. Boghosian, Phys. Rev. E 62, 8282 (2000).

[44] M. Nekovee and P. V. Coveney, J. Am. Chem. Soc. 123, 12380 (2001).

[45] G. Giupponi, J. Harting, and P. V. Coveney, Europhys. Lett. 73, 533 (2006).

[46] N. González-Segredo and P. V. Coveney, Europhys. Lett. 65, 795 (2004).

[47] S. M. Pickles et al., in Proceedings of the Workshop on Case 
Studies on Grid Applications at GGF 10, 2004.

[48] J. Harting, M. Harvey, J. Chin, and P. V. Coveney, Comput. Phys. Commun. 165, 97 (2004).

[49] J. Harting, M. Harvey, J. Chin, M. Venturoli, and P. V. Coveney, Philos. Trans. R. Soc. London, Ser. A 363, 1895 (2005).

[50] J. Chin and P. V. Coveney, Philos. Trans. R. Soc. London, Ser. A 462, 3575 (2006).

[51] N. González-Segredo, J. Harting, G. Giupponi, and P. V. Coveney, Phys. Rev. E 73, 031503 (2006).

[52] P. J. Higuera, S. Succi, and R. Benzi, Europhys. Lett. 9, 345 (1989).

[53] P. L. Bhatnagar, E. P. Gross, and M. Krook, Phys. Rev. 94, 511 (1954).

[54] S. Chen, H. Chen, D. Martínez, and W. Matthaeus, Phys. Rev. Lett. 67, 3776 (1991).

[55] X. Shan and H. Chen, Phys. Rev. E 47, 1815 (1993).

[56] X. Shan and H. Chen, Phys. Rev. E 49, 2941 (1994).

[57] M. Nekovee and P. V. Coveney, J. Am. Chem. Soc. 123, 12380 (2001).

[58] A. Lees and S. Edwards, J. Phys. C 5, 1921 (1972).

[59] J. Harting, M. Venturoli, and P. V. Coveney, Philos. Trans. R. Soc. London, Ser. A 362, 1703 (2004).

[60] P. J. Love, P. V. Coveney, and B. M. Boghosian, Phys. Rev. E
64, 021503 (2001).

[61] D. Rothman, Europhys. Lett. 14, 337 (1991).

[62] A. H. Krall, J. V. Sengers, and K. Hamano, Phys. Rev. Lett. 69, 1963 (1992).

[63] T. Ohta, H. Nozaki, and M. Doi, J. Comput. Phys. 93, 2664 (1990).

[64] F. Corberi, G. Gonnella, and A. Lamura, Phys. Rev. E 62, 8064 (2000).

[65] T. Hashimoto, K. Matsuzaka, E. Moses, and A. Onuki, Phys. Rev. Lett. 74, 126 (1994).

[66] Z. Shou and A. Chakrabarti, Phys. Rev. E 61, R2200 (2000).

[67] F. Corberi, G. Gonnella, and A. Lamura, Phys. Rev. Lett. 81, 3852 (1998).

[68] F. Corberi, G. Gonnella, and A. Lamura, Phys. Rev. Lett. 83, 4057 (1999).

[69] F. Corberi, G. Gonnella, and A. Lamura, Phys. Rev. E 66, 016114 (2002).

[70] F. Corberi, G. Gonnella, and D. Suppa, Phys. Rev. E 63, 040501(R) (2001).

[71] A. H. Krall, J. V. Sengers, and K. Hamano, Phys. Rev. E 48, 357 (1993).

[72] K. Matsuzaka, H. Jinnai, T. Koga, and T. Hashimoto, Macromolecules 30, 1146 (1997). 